\title{
Papers
}

\section{Cost effectiveness analysis of intensive versus conventional follow up after curative resection for colorectal cancer}

\author{
Andrew G Renehan, Sarah T O’Dwyer, David K Whynes
}

\begin{abstract}
Objective To determine the cost effectiveness of intensive follow up compared with conventional follow up in patients with colorectal cancer.

Design Incremental cost effectiveness analysis recognising differences in follow up strategies, based on effectiveness data from a meta-analysis of five randomised trials.

Setting United Kingdom.

Main outcome measures Taking a health service perspective, estimated incremental costs effectiveness ratios for each life year gained for five trials and four trials designed for early detection of extramural recurrences (targeted surveillance). Results Based on five year follow up, the numbers of life years gained by intensive follow up were 0.73 for the five trial model and 0.82 for the four trial model. For the five trials, the adjusted net (extra) cost for each patient was $£ 2479$ (€3550; \$4288) and for each life year gained was $£ 3402$, substantially lower than the current threshold of NHS cost acceptability ( $£ 30000)$. The corresponding values for the four trial model were £2529 and $£ 3077$, suggesting that targeted surveillance is more cost effective. The main predictor of incremental cost effectiveness ratios was surveillance costs rather than treatment costs. Judged against the NHS threshold of cost acceptability, the predicted incremental cost threshold was ninefold and the effectiveness threshold was $3 \%$.

Conclusions Based on the available data and current costs, intensive follow up after curative resection for colorectal cancer is economically justified and should be normal practice. There is a continuing need to evaluate the efficacy of specific surveillance tools: this study forms the basis for economic evaluations in such trials.
\end{abstract}

\section{Introduction}

More than 35000 new cases of colorectal cancer occur in the United Kingdom each year, representing a major disease burden on health services. ${ }^{12}$ At initial presentation, around two thirds of patients undergo resection with curative intent, and most enter some type of long term follow up. ${ }^{3}$ The rationale behind this is threefold: psychological support, facilitation of audit, and the early detection and treatment of recurrent disease, with potential improvement in survival. ${ }^{4}$ The merits of early detection and treatment of recurrent disease have been vigorously debated. Recently, the present authors and a Cochrane review group independently reported two meta-analyses of all randomised trials of follow up strategies for patients treated for colorectal cancer and showed a significant improvement in all cause mortality in patients followed intensively. ${ }^{56} \mathrm{~A}$ further ran- domised trial has since been published supporting these findings. ${ }^{7}$ These data are the first direct evidence that intensive follow up improves survival.

Against the emerging evidence of the effectiveness of intensive follow up, follow up practice varies widely worldwide and, among these many different protocols, the costs to health services are considerable. ${ }^{8-10}$ We compared the cost effectiveness of intensive follow up with conventional follow up in patients treated for colorectal cancer.

\section{Methods}

Our cost effectiveness analysis was based on the results of our previous meta-analysis of five randomised trials (1342 patients; five trial model), which showed a significant reduction in all cause mortality at five years (absolute reduction 7\%) in patients followed intensively. ${ }^{51-15}$ Our second analysis was based on effectiveness data from four trials (four trial model), where surveillance tools were targeted at detecting of extraluminal recurrences and where improvement in survival was more pronounced (absolute reduction 9\%). ${ }^{11-14}$

\section{Costs}

We adopted a health service perspective and calculated patient specific costs related to five year follow up strategies for colorectal cancer (intensive follow up compared with conventional follow up) by estimating costs for surveillance and treatment of recurrences. Costs were based on 2002 UK prices and included direct, indirect, and overhead costs. We used a "bottom-up" approach, rounding costs upward and seeking to be as inclusive as possible in estimates. Sources included the Department of Health reference costs and related cost effectiveness studies (see bmj.com). ${ }^{16-18}$ The Department of Health reference costs are aggregate direct costs for trust hospitals nationwide and present costs as mean, minimum, and maximum values.

Because follow up regimens and surveillance tests varied considerably between and within trials, we estimated costs separately for each trial and then calculated the costs for each patient. ${ }^{5}$ Costs included clinical follow up visits (administration, clinical examination, consumables), laboratory tests (reagents, consumables, staff, service costs, processing and reporting), endoscopic investigations and polypectomy (administration, drugs, consumables, staff, sterilisation, annual equivalent costs for equipment), and radiology (staff, consumables, service costs,

Additional information and a file for calculation of estimates are on $\mathrm{P}+$ bmj.com 
Table 1 Unit cost estimate for surveillance and treatment of recurrent colorectal cancer

\begin{tabular}{|c|c|c|}
\hline Costs & Mean cost $(£)$ & Maximum cost $(£)$ \\
\hline \multicolumn{3}{|l|}{ Surveillance } \\
\hline Physical examination & 58.80 & 281.80 \\
\hline \multicolumn{3}{|l|}{ Laboratory tests: } \\
\hline Full blood count & 9.30 & 18.30 \\
\hline Erythrocyte sedimentation rate & 9.30 & 18.30 \\
\hline Liver function test & 9.30 & 23.30 \\
\hline Carcinoembryonic antigen & 9.30 & 23.30 \\
\hline Faecal occult blood test & 14.00 & 76.00 \\
\hline \multicolumn{3}{|l|}{ Endoscopy: } \\
\hline Rigid sigmoidoscopy & 98.80 & 321.80 \\
\hline Flexible sigmoidoscopy (examination only) & \multirow[b]{2}{*}{156.41} & \multirow[b]{2}{*}{578.37} \\
\hline $\begin{array}{l}\text { Flexible sigmoidoscopy with biopsy or } \\
\text { polypectomy* }\end{array}$ & & \\
\hline Colonoscopy (examination only) & \multirow{2}{*}{229.15} & \multirow{2}{*}{545.92} \\
\hline Colonoscopy with biopsy or polypectomy $†$ & & \\
\hline \multicolumn{3}{|l|}{ Imaging: } \\
\hline Chest radiography & 22.40 & 148.40 \\
\hline Barium enema radiography & 138.40 & 610.40 \\
\hline Liver ultrasonography & 104.40 & 264.40 \\
\hline Abdominal or pelvic computed tomography & 171.20 & 777.20 \\
\hline \multicolumn{3}{|l|}{ Treatment } \\
\hline \multicolumn{3}{|l|}{ Intensive follow up: } \\
\hline Local salvage therapy & 11258 & 32108 \\
\hline Local palliative therapy & 4102 & 14284 \\
\hline Hepatic salvage therapy & 9513 & 2541 \\
\hline Hepatic palliative therapy & 1288 & 6075 \\
\hline \multicolumn{3}{|l|}{ Control follow up: } \\
\hline Local salvage therapy & 11630 & 33915 \\
\hline Local palliative therapy & 4377 & 15622 \\
\hline Hepatic salvage therapy & 9885 & 27348 \\
\hline Hepatic palliative therapy & 1563 & 7413 \\
\hline
\end{tabular}

$£ 1(€ 1.4 ; \$ 1.7)$.

Estimates include direct, indirect, and overhead costs based on 2002 prices. Sources of cost estimates from Department of Health reference costs, Gilbert et al, ${ }^{17}$ multicentre aneurysm screening study, ${ }^{18}$ and in-house financial department (see bmj.com).

${ }^{*}$ Modelled that $21 \%$ of patients may have distal colonic polyps ${ }^{19}$; probably an overestimate but included as part of "bottom-up" approach to estimations.

tModelled that $27 \%$ of patients undergoing surveillance colonoscopy will require biopsy or polypectomy (control data from Sandler et a ${ }^{20}$ ).

processing and reporting, annual equivalent costs for equipment; table 1). ${ }^{19}{ }^{20}$ As follow up rates were high for all trials, we modelled full attendance for each prescribed examination and surveillance investigation until five years or death. ${ }^{5}$ We modelled for additional investigations (namely computed tomography) owing to a $15 \%$ false positive rate when testing for serum carcinoembryonic antigen. ${ }^{21}$

In calculating treatment costs, we incorporated into our model three possible outcomes after the detection of recurrences: inoperable disease requiring symptomatic treatment and palliative care, salvage with cure, and salvage with subsequent failure and palliative care (see figure on bmj.com). For patients followed conventionally, we modelled that the initial assessment of recurrent disease (the decision between palliation and salvage) was based on off-trial investigations, whereas the assessment of recurrent disease was encompassed by protocol investigations in patients followed intensively. ${ }^{14}$ Treatment costs were then estimated for salvage and palliative treatments for local, hepatic, and other site recurrences. Salvage costs included surgery with average hospital stay, perioperative investigations, high dependency care, blood transfusion, drug usage, stoma care, and care by a district nurse (see bmj.com). A further $35 \%$ was added to salvage therapy costs (intensive and conventional follow up) to account for high rates of complications and reoperations associated with these types of treatments. ${ }^{22}{ }^{23}$ Palliation costs included palliative surgery and stoma care, palliative radiotherapy, palliative medical care, and cancer care in the community. Chemotherapy regimens were not used in any trial.

\section{Outcome measures}

The primary outcome was the extra cost per unit of outcome obtained in comparing one treatment with another (incremental cost effectiveness ratio) for each change in life year for intensive follow up compared with conventional follow up. ${ }^{24}$ We calculated life years lost and gained for each trial using average life expectancies for the UK population and taking into account the proportions of males and females, the mean ages at initial treatment, and the number of deaths. ${ }^{25}$ Costs were calculated for each trial and then expressed as costs for each patient and aggregated. The probability that intensive follow up is cost effective depends on how much the NHS is willing to pay for each life year gained-the value of $£ 30000$ ( $€ 42968 ; \$ 51888$ ) was used (referred to as NHS cost acceptability), reflecting the currently perceived threshold in the United Kingdom. ${ }^{26}$

\section{Censoring and discounting}

In the absence of individual patient data, we modelled the distribution of censored events (deaths) by constant numbers for each three month period. In practice, surveillance would generally be discontinued (censored) after recurrence, such that this model tends to overestimate surveillance costs. In the base case analysis, we discounted benefit effects at $1.5 \%$ and costs at $6.0 \% .{ }^{27}$

\section{Sensitivity analyses}

We undertook several sensitivity analyses to illustrate the impact of the principal aspects of uncertainty on the estimate of cost effectiveness. These included, separately and together, changes to discount rates as proposed by the US Panel on Cost Effectiveness in Health and Medicine, ${ }^{24}$ distributions of deaths (constant number, constant rate, and all deaths at end of five years), false positive test rates, and maximum surveillance and treatment costs.

Using the NHS cost acceptability of $£ 30000$ as reference, we calculated the incremental increase in costs required to increase the incremental cost effectiveness ratio to the limit of NHS cost acceptability (incremental cost threshold) by simultaneous incremental increases in surveillance and treatment costs for both intensive and conventional follow up. We calculated the lowest level of effectiveness-absolute reduction in mortality-required to increase the incremental cost effectiveness ratio to the limit of NHS cost acceptability (threshold of effectiveness). The threshold of effectiveness was calculated by altering differences in benefit between follow up strategies by amounts equivalent to $1 \%$ change in mortality (see bmj.com).

\section{Results}

\section{Cost effectiveness}

The figure shows the Forest plot for all cause mortality with absolute reductions in mortality, together with the calculated life years lost and gained for each patient for each study and associated incremental cost effectiveness ratios. For the five trial model, the lost and gained calculations favoured intensive follow up by 0.73 life years gained for each patient, increasing to 0.82 life years gained for each patient for the four trial model, consistent with the observations from our previous meta-analysis. ${ }^{5}$ In the five trial model, the adjusted net (extra) cost for each patient was $£ 2479$ and for each life year gained was £3402, substantially lower than the current NHS threshold of cost acceptability. The 


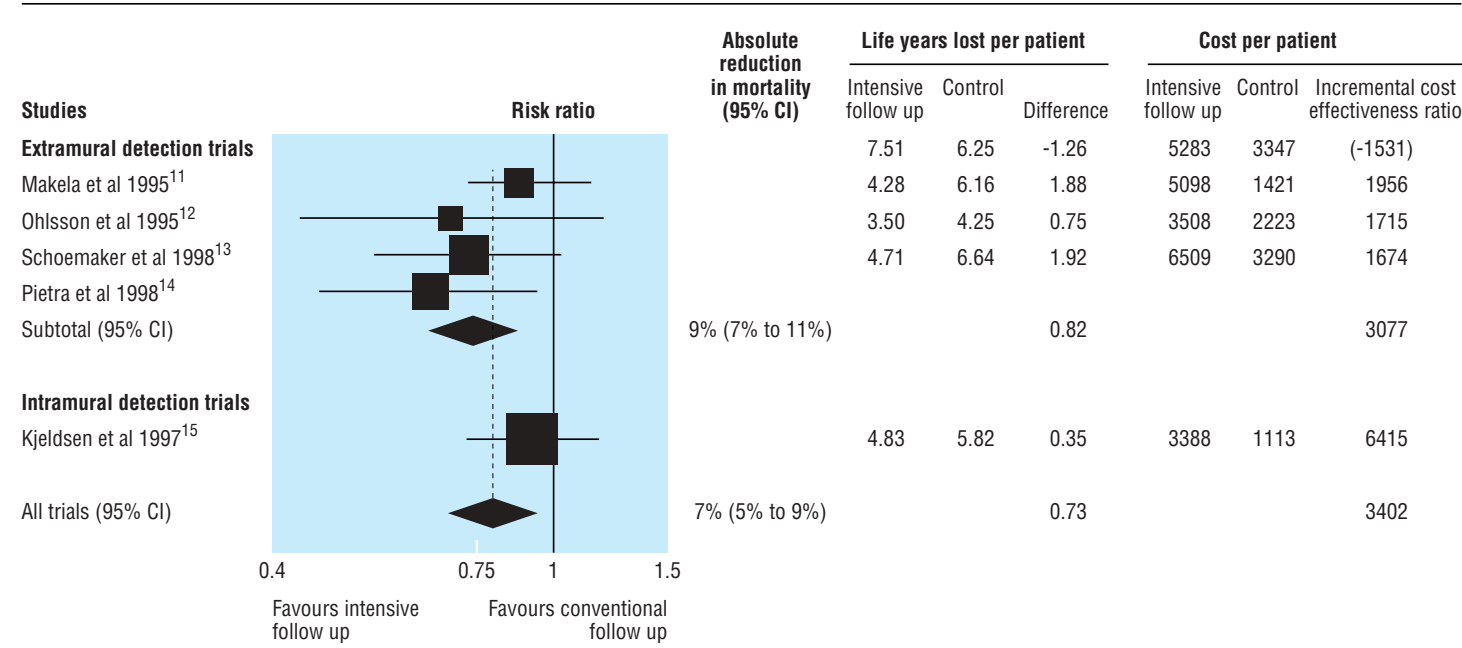

Forest plot of randomised trials. Pooled analysis with summary estimates (Mantel-Haenszel fixed effects method) are for all cause mortality (adapted from Renehan et al $2002^{5}$ ). Data are categorised into extramural and intramural detection groups. Positive difference indicates time gained (improved survival). See bmj.com for calculation of estimates. Incremental cost effectiveness ratio=[costs for each patient for intensive follow up minus control follow up]/[discounted life years either gained or lost]

corresponding values for the four trial model were $£ 2529$ and $£ 3077$, suggesting that targeted surveillance is more cost effective.

Changes in benefit and discount rates and in false positive test rates made little impact on the incremental cost effectiveness ratios in both models (data for four trial model not shown; table 2). Changing the distributions of deaths yielded similar incremental cost effectiveness ratios, suggesting that the assumptions within the primary models were robust. Worst case scenario analyses showed that substituting with maximum surveillance costs produced greater increases in incremental cost effectiveness ratios compared with substituting with maximum treatment costs, indicating that surveillance cost is the most important determinant of cost effectiveness. All incremental cost effective- ness ratios for maximum cost estimates fell within the NHS cost acceptability threshold. The incremental cost threshold was near ninefold for both the five trial (£30 620) and four trial (£27 695) models. For both models the threshold of cost effectiveness was an absolute reduction in mortality of $3 \%$.

\section{Discussion}

Intensive follow up after curative resection for colorectal cancer improves all cause mortality at five years and has a cost effectiveness ratio well within the NHS acceptability of willingness to pay for each life year gained. This economic analysis, based on effectiveness data from a previous meta-analysis, provides firm

Table 2 Sensitivity analyses

Costs per patient (£)

\begin{tabular}{|c|c|c|c|c|}
\hline \multirow[b]{2}{*}{ Model } & & \multirow[b]{2}{*}{ Extra costs } & \multirow[b]{2}{*}{ Incremental cost effectiveness ratio } \\
\hline & Intensive follow up & Control & & \\
\hline \multicolumn{5}{|l|}{ Five trial model } \\
\hline 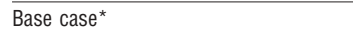 & 4758 & 2279 & 2479 & 3402 \\
\hline \multicolumn{5}{|l|}{ Different discounts: } \\
\hline Undiscounted & 5173 & 2417 & 2756 & 3540 \\
\hline Benefits and costs discounted at $3 \%$ & 4953 & 2344 & 2609 & 3832 \\
\hline \multicolumn{5}{|l|}{ Distribution of death: } \\
\hline Constant rate & 4721 & 2255 & 2467 & 3285 \\
\hline All deaths at end of five years & 5160 & 2471 & 2689 & 4244 \\
\hline \multicolumn{5}{|l|}{ False positive test rate: } \\
\hline Zero rate (no false positives) & 4757 & 2279 & 2479 & 3402 \\
\hline Rate at $30 \%$ & 4758 & 2279 & 2479 & 3403 \\
\hline \multicolumn{5}{|l|}{ Maximum costs: } \\
\hline Surveillance & 12310 & 5033 & 7277 & 9989 \\
\hline Treatment & 8874 & 5836 & 3038 & 4171 \\
\hline Surveillance and treatment & 16426 & 8589 & 7837 & 10757 \\
\hline \multicolumn{5}{|l|}{ Four trial model (targeted) } \\
\hline${\text { Base } \text { case }^{\star}}$ & 5100 & 2570 & 2529 & 3077 \\
\hline \multicolumn{5}{|l|}{ Maximum costs: } \\
\hline Surveillance & 13403 & 5917 & 7486 & 9108 \\
\hline Treatment & 9509 & 6390 & 3119 & 3794 \\
\hline Surveillance and treatment & 17813 & 9737 & 8076 & 9825 \\
\hline
\end{tabular}

$£ 1(€ 1.4 ; \$ 1.7)$.

Estimates are costs for each patient followed, assuming all patients started follow up.

${ }^{*}$ Survival analysis at constant number of deaths for each time period, false positive test rate at $15 \%$, benefit effect discount at $1.5 \%$, and cost discount at $6 \%$. 
estimates of the cost of intensive follow up. The data also show that targeted follow up improves cost effectiveness.

\section{Strengths compared with other studies}

Previous studies have examined economic issues related to follow up after treatment for colorectal cancer, ${ }^{28-33}$ but predated the effectiveness data provided by the two recently published meta-analyses. ${ }^{5}{ }^{6}$ The present analysis is strengthened by the inclusion of a comprehensive search to include many aspects of cost estimates (a "bottom-up" approach) and sensitivity analyses at several levels. Further strengths were the robustness of the results: adjustments to maximise costs yielded incremental cost effectiveness ratios well within the threshold for NHS cost acceptability, the incremental cost threshold was high (ninefold increases), and the thresholds of effectiveness were $3 \%$ for both models.

Our study emphasises a further aspect, that around equal proportions (one third) of patients with colorectal cancer develop recurrences, irrespective of follow up strategy. After detection of recurrence, palliative therapy (more common in control patients) is not without expense and partially offsets the costs of salvage therapy (more common in patients intensively followed).

\section{Study limitations}

The first limitation of our study was the clinical heterogeneity of follow up regimens among the five trials. ${ }^{5}$ No study directly compared specific tests, and therefore it was not possible to evaluate the cost effectiveness of specific surveillance tools. A second limitation was the lack of quality of life data for follow up of patients with colorectal cancer, although one study has assigned a quality adjusted life year of 0.83 based on data from a chemotherapy trial. ${ }^{29}$ As intensive follow up does not significantly raise patient anxieties, this may prove a reasonable estimate, but further studies are required before these values can be incorporated into health economic calculations. ${ }^{34}$ The third limitation was the absence of individual patient data, such that we had to make assumptions about survival and recurrence patterns. These assumptions, however, held firm, as shown by the sensitivity analysis. The fourth limitation was that the included trials were conducted over a decade ago, and thus did not include aspects of contemporary practice (for example, multidisciplinary management) that could impact on costs or effectiveness. The final limitation is that we were unable to compare intensive follow up with absolute no follow up (zero surveillance costs), although in modern practice the acceptability of a no follow up strategy is questionable. $^{35}$

\section{Implications for health policy}

The strategy of intensive follow up after curative resection for colorectal cancer is economically justified and should be normal practice. As a screening type health strategy, intensive follow up of patients with colorectal cancer compares favourably with screening for breast and colorectal cancers and for abdominal aortic aneurysms. ${ }^{183637}$ Moreover, compared with population screening, these patients are self defined, well motivated, and compliant. $^{38}$

\section{Factors that could affect future results}

Large randomised trials are needed to evaluate the efficacy of specific surveillance tools. ${ }^{55}$ These studies must include cost effectiveness analyses and quality of life assessments, in addition to other issues that were beyond the scope of our analysis. Firstly, costs beyond five years after initial treatment need to be considered as a proportion of patients with recurrences undergoing salvage therapy will have delayed second recurrences. Secondly,

\section{What is already known on this topic}

Intensive follow up after curative resection for colorectal cancer improves survival (absolute effects 7-9\%)

The cost effectiveness of intensive surveillance is uncertain

\section{What this study adds}

The number of years gained through intensive surveillance over five years is 0.73 to 0.82 for each patient

The adjusted net cost for each patient was £2479 and for each life year gained was $£ 3402$, substantially lower than the NHS threshold of cost acceptability ( $£ 30000$ )

Corresponding values for four trials using targeted surveillance were $£ 2529$ and $£ 3077$, suggesting that this approach may be more expensive but improves cost effectiveness

the included trials predated the widespread use of chemotherapy for advanced colorectal cancer or as an adjunct to salvage reoperation, approaches that may improve survival. ${ }^{39}$ This potential improvement in effectiveness will be coupled with increased costs. However, as maximising treatments costs (without changes in surveillance costs) had only minor effects on incremental cost effectiveness ratios, it is tempting to speculate that chemotherapy, expensive when considered in selected groups, may be cost effective in the wider context. ${ }^{39}$ Thirdly, our analysis treated local, hepatic, and other site recurrences as mutually exclusive events. In practice there is overlap, which will tend to attenuate costs. Fourthly, we did not address societal perspectives, such as travel and time off work, which are important determinants of compliance in screening for colorectal cancer and may thus be relevant in cancer surveillance..$^{40}$ Fifthly, it was not possible to estimate capital costs for additional laboratory and clinical capacity. Finally, we did not consider the benefits of intensive follow up from factors other than salvage for recurrent disease-for example, psychosocial or improved treatment of coincidental disease. Evidence from our meta-analysis shows that some reductions in all cause mortality associated with intensive follow up are attributable to factors other than salvage therapy (unpublished data). ${ }^{5}$

Contributors: AGR and STO'D initiated the design of the study. AGR and DKW undertook the economic analyses. All authors contributed to the writing of the final draft of the manuscript. STO'D will act as guarantor for the paper.

Funding: None.

Competing interests: None declared.

Ethical approval: Not required.

1 Office of National Statistics. Cancer statistics: registrations, series MB1 for 1999. www.statistics.gov.uk (accessed 18 Mar 2003).

2 Information and Statistics Division (ISD) Scotland. Cancer: facts and figures for 1999. www.show.scot.nhs.uk/isd (accessed 18 Mar 2003).

Kievit J, Bruinvels DJ. Detection of recurrence after surgery for colorectal cancer. Eur J Cancer 1995;31a:1222-5.

4 Scholefield JH, Steele RJ. Guidelines for follow up after resection of colorectal cancer. Gut 2002;51(Suppl 5):V3-5.

5 Renehan AG, Egger M, Saunders MP, O'Dwyer ST. Impact on survival of intensive follow up after curative resection for colorectal cancer: systematic review and meta-analysis of randomised trials. BMJ 2002;324:813

6 Jeffrey GM, Hickey BE, Hider P. Follow-up strategies for patients treated for non-metastatic colorectal cancer. In: Cochrane Library, Issue 1. Oxford: Update Software, 2002.

7 Secco GB, Fardelli R, Gianquinto D, Bonfante P, Baldi E, Ravera G, et al. Efficacy and cost of risk-adapted follow-up in patients after colorectal cancer surgery: a prospective, randomized and controlled trial. Eur J Surg Oncol 2002;28:418-23. 
8 Mella J, Datta SN, Biffin A, Radcliffe AG, Steele RJ, Stamatakis JD. Surgeons' follow-up practice after resection of colorectal cancer. Ann R Coll Surg Engl 1997;79:206-9.

9 Bruinvels DJ, Stiggelbout AM, Klaassen MP, Kievit J, Dik J, Habbema F, et al. Follow-up after colorectal cancer: current practice in the Netherlands. Eur $J$ Surg $1995 ; 161: 827-31$.

10 Virgo KS, Wade TP, Longo WE, Coplin MA, Vernava AM, Johnson FE. Surveillance after curative colon cancer resection: practice patterns of surgical subspecialists. Ann Surg Oncol 1995;2:472-82.

11 Makela JT, Laitinen SO, Kairaluoma MI. Five-year follow-up after radical surgery for colorectal cancer. Results of a prospective randomized trial. Arch Surg 1995:130:1062-7.

12 Ohlsson B, Breland U, Ekberg H, Graffner H, Tranberg KG. Follow-up after curative surgery for colorectal carcinoma: randomized comparison with no follow-up. Dis Colon Rectum 1995.38:619-26.

13 Schoemaker D, Black R, Giles L, Toouli J. Yearly colonoscopy, liver CT, and chest radiography do not influence 5-year survival of colorectal cancer patients. Gastroenterology 1998;114:7-14.

14 Pietra N, Sarli L, Costi R, Ouchemi C, Grattarola M, Peracchia A. Role of follow-up in management of local recurrences of colorectal cancer: a prospective, randomized study. Dis Colon Rectum 1998;41:1127-33.

15 Kjeldsen BJ, Kronborg O, Fenger C, Jorgensen OD. A prospective randomized study of follow-up after radical surgery for colorectal cancer. Br J Surg 1997;84:666-9.

16 Department of Health NHS reference costs. www.doh.gov.uk/nhsexec/refcosts.htm (accessed 18 Mar 2003).

17 Gilbert RE, Augood C, Gupta R, Ades AE, Logan S, Sculpher M, et al. Screening for Down's syndrome: effects, safety, and cost effectiveness of first and second trimester strategies. BMJ 2001;323:423-5.

18 Multicentre aneurysm screening study (MASS): cost effectiveness analysis of screening for abdominal aortic aneurysms based on four year results from randomised controlled trial. BMJ 2002;325:1135

19 UK Flexible Sigmoidoscopy Screening Trial Investigators. Single flexible sigmoidoscopy screening to prevent colorectal cancer: baseline findings of a UK multicentre randomised trial. Lancet 2002;359:1291-300.

20 Sandler RS, Halabi S, Baron JA, Budinger S, Paskett E, Keresztes R, et al. A randomized trial of aspirin to prevent colorectal adenomas in patients with previous colorectal cancer. N Engl J Med 2003;348:883-90.

21 Kievit J. Follow-up of patients with colorectal cancer: numbers needed to test and treat. Eur J Cancer 2002;38:986-99.

22 Fusai G, Davidson BR. Management of colorectal liver metastases. Colorectal Dis 2003;5:2-23.

23 Wanebo HJ, Antoniuk P, Koness RJ, Levy A, Vezeridis M, Cohen SI, et al. Pelvic resecion of recurrent rectal cancer: technical considerations and outcomes. Dis Colo Rectum 1999;42:1438-48.

24 Gold MR, Siegel JE, Russell LB, Weinstein MC. Cost-effectiveness in health and medicine. New York: Oxford University Press, 1996

25 UK Government Actuary's Department. Life tables. www.gad.uk/policy and stats life_tables.htm (accessed 18 Mar 2003).

26 Raftery J. NICE: faster access to modern treatments? Analysis of guidance on health technologies. BMJ 2001;323:1300-3.

27 Torgerson D, Raftery J. Economics notes: discounting. BMJ 1999;319:914-5.
28 Virgo KS, Vernava AM, Longo WE, McKirgan LW, Johnson FE. Cost of patient follow-up after potentially curative colorectal cancer treatment. JAMA 1995;273:183741

29 Norum J, Olsen JA. A cost-effectiveness approach to the Norwegian follow-up programme in colorectal cancer. Ann Oncol 1997;8:1081-7.

30 Edelman MJ, Meyers FJ, Siegel D. The utility of follow-up testing after curative cance therapy: a critical review and economic analysis. J Gen Intern Med 1997;12:318-31.

31 Graham RA, Wang S, Catalano PJ, Haller DG. Postsurgical surveillance of colon cancer: preliminary cost analysis of physician examination, carcinoembryonic antigen testing. chest x-ray, and colonoscopy. Ann Surg 1998;228:59-63.

32 Beart RW Jr. Follow-up: does it work? Can we afford it? Surg Oncol Clin N Am 2000;9:827-34.

33 Van den Hout WB, van den Brink M, Stiggelbout AM, van de Velde CJH, Kievit J. Costeffectiveness analysis of colorectal cancer treatments. Eur J Cancer 2002;38:953-63.

34 Stiggelbout AM, de Haes JC, Vree R, van de Velde CJ, Bruijninckx CM, van Groningen $\mathrm{K}$, et al. Follow-up of colorectal cancer patients: quality of life and attitudes towards follow-up. Br J Cancer 1997;75:914-20.

35 Renehan AG, Egger M, Saunders MP, Whynes D, O'Dwyer ST. Intensive follow-up after colorectal cancer. Recent successes and future challenges. In: Cunningham D, Topham C, Miles A, eds. The effective management of colorectal cancer, 4th ed. London: Aesculapius Medical Press, 2004.

36 Wang H, Karesen R, Hervik A, Thoresen SO. Mammography screening in Norway: results from the first screening round in four counties and cost-effectiveness of a modeled nationwide screening. Cancer Causes Control 2001;12:39-45.

37 Whynes DK, Neilson AR, Walker AR, Hardcastle JD. Faecal occult blood screening for colorectal cancer: is it cost-effective? Health Econ 1998;7:21-9.

38 Kjeldsen BJ, Thorsen H, Whalley D, Kronborg O. Influence of follow-up on health-related quality of life after radical surgery for colorectal cancer. ScandJ Gastroenterol 1999;34:509-15.

39 National Institute for Clinical Excellence. A review of the evidence for the clinical and cost-effectiveness of ironotecan, oxaliplatin and raltitrexed for the treatment of advanced colorectal cancer. www.nice.org.uk (accessed 18 Mar 2003).

40 Frew E, Wolstenholme JL, Atkin W, Whynes DK. Estimating time and travel costs incurred in clinic based screening: flexible sigmoidoscopy screening for colorectal cancer. J Med Screen 1999;6:119-23.

(Accepted 16 October 2003)

bmj.com 2004;328:81

Department of Surgery, Christie Hospital NHS Trust, Withington, Manchester M20 4BX

Andrew G Renehan senior research fellow

Sarah T O'Dwyer director

Department of Economics, University of Nottingham, Nottingham NG7 2RD David K Whynes professor

Correspondence to: A G Renehan

arenehan@picr.man.ac.uk 